\title{
A Schema-Based Study of Interpreting Process
}

\author{
Yu Jun \\ Faculty of International Languages, Xiamen University of Technology \\ Xiamen, China
}

\begin{abstract}
Interpretation has been examined from various perspectives with fruitful results. But the study of interpretation from a schematic approach, despite its bright prospect, is relatively rare. Interpretation is a knowledge-based activity, and schemata is an efficient representation form of knowledge. In this paper, five types of schema in the interpreting process, namely language schema, topic schema, text schema, cultural schema and strategy schema are identified as crucial for the interpreter to bring about successful communication. A schema model for interpreting process is put forward to illustrate how these types of schema work together towards successful interpretation. From the perspective of mental efforts in interpretation, the roles of these schemata in interpretation, and their efficient deployment to achieve coordinated distribution of mental efforts are examined, which further revealing the importance of schemata in interpretation.
\end{abstract}

\section{Keywords—schema; model; interpreting; process}

\section{INTRODUCTION}

In the age of global intercultural communication, interpreting is playing an increasingly important role in modern society. Interpreting has entered a golden age in China and so have interpreting studies, to which a growing number of brilliant researchers are dedicated. Interpreting research in China, though still an emerging discipline to be further explored and strengthened, has made fruitful progress with models for interpreting put forward, insights gained, and new approaches developed. Among these approaches is the examination of the nature and process of interpreting from the perspective of cognitive science, such as cognitive psychology and cognitive linguistics. The implication of cognitive science in interpreting studies has been duly recognized but not fully explored, however.

The paper is an attempt to look into the interpreting process from the cognitive perspective, or rather, to be more specific, from that of schema (plural, schemata) which has been applied in various fields such as Artificial Intelligence (AI), discourse analysis, and EFL teaching where a great deal of studies have been done on schemata's role in reading comprehension and listening comprehension with insightful results. Schemata's application to interpreting studies, however, has been relatively rare despite its bright prospect.

The author, in this paper, ventures into a schematic study of interpretation with view to what a role the schema plays in interpretation as a knowledge-based activity and an act of intercultural communication and what insights it may offer to the improvement of interpreting performance.

\section{SCHEMATIC REPRESENTATION OF KNOWLEDGE}

\section{A. Interpretation as a Knowledge-based Activity}

In the interpreting process, the knowledge base of the interpreter plays a fundamental role. According to Gile [1], the knowledge base can be divided into two sub-branches, namely linguistic knowledge (LK) and extra-linguistic knowledge (ELK), which are dependent on and complementary to each other in the interpreting process. Without a strong knowledge base, it is hard for the interpreter to achieve successful interpreting, as he or she has to rely on his or her knowledge base to decode the speaker's messages and to reformulate them in the target language. The need for an efficient utilization of linguistic knowledge and extra-linguistic knowledge is felt throughout the interpreting process. Interpretation is in fact a knowledge-based activity.

\section{B. Schematic Knowledge Representation}

The notion of schema is generally associated with the early work on story recall by Bartlett [2]. Bartlett conducted experiments conducive to memory errors, so that he could examine the guiding function of schemata in the reconstruction process. Minsky [3] uses the term "frame" to describe schemata related to specific occasions. And one may find the term "schema" used in text-generation theory to mean only "rhetorical techniques" [4].

In ELT it is often said that there are two types of schema: formal and content. Formal schemata [5] are described as abstract, encoded, internalized, coherent patterns of metalinguistic, discoursal, and textual organization (e.g., rhetorical patterns, story grammar, narrative scripts) that guide expectations in our attempts to understand a meaningful piece of language. Content schemata are less abstract and must presumably be about the physical world of discernible objects and actions.

While in discourse analysis, Cook [6] classified schema into "language schema", "word schema" and "text schema" referring to language knowledge, knowledge of the world and of certain text types respectively.

In information processing, schemata are often employed to infer from what's left unsaid through insertion of default values For example, if you are asked to visualize the type of balls from the following sentences: 
(a) Tiger Woods hit the ball.

(b) Deng Yaping hit the ball.

(c) Venus and Serena Williams hit the ball.

Are your answers: (a) golf ball, (b) ping-pong ball, and (c) tennis ball? If so, where did you get the ideas for your answers? It is your topic schema that informed you that (a) Tiger Woods is a golf pro, (b) Deng Yaping is a ping-pong player, and (c) the Williams sisters are professional tennis players. If you were unable to guess the type of balls, you probably are not a sports fan and do not have the appropriate schema to associate the names with the type of balls.

In cognitive science, it is of great importance to find out about the structure, retrieval, and activation, modification and acquisition of knowledge in human mind for man to acquire, organize and utilize knowledge more efficiently. Thus hypothetical models of knowledge representation have been set up with working mechanism of such models suggested; schema, which can represent both declarative knowledge and procedural knowledge, is maybe one of the most powerful and useful.

\section{FIVE TYPES OF SCHEMA INVOLVED IN THE INTERPRETING PROCESS}

Interpretation is "the oral transfer of a message from one language into another" [7]. To make the transfer successful, the interpreter is supposed to have a good command of linguistic knowledge and extra-linguistic knowledge as well, in addition to mastery of interpreting skills and awareness of cultural difference. The interpreting process is beyond doubt a very complex one involving many factors. And it has been looked into from different approaches such as that of communication. Schemata are involved in interpreting which is a knowledge-based activity.

In the interpreting process, five types of schema, namely, language schema, topic schema, text schema, cultural schema and strategy schema, are identified by the author as crucial for the interpreter to bring about successful communication between people who speak a different language.

\section{A. Language Schema}

For an interpreter to achieve successful interpreting, language competence is a vital part of what is required of him or her. From the schematic perspective, his or her language knowledge can be referred to as language schema, which can be further divided into phonological schema, syntax schema, semantic schema, and pragmatic schema. Phonological schema refers to the interpreter's phonological knowledge that enables him or her to discern meaningful human sounds, link them to their lexical meaning, and pronounce them as well. Syntax schema refers to the interpreter's syntactic knowledge of a language, namely, the rules of forming sentences in a language by combining words, word groups and phrases. Semantic schema refers to the interpreter's semantic knowledge, knowledge of determining meaning conveyed in a discourse at the word, grammatical and contextual level as well rather than at the word level alone, so as to make the right choice of expression in the target language. Pragmatic schema refers to the interpreter's pragmatic knowledge, or in other words, knowledge of "use of language in human communication as determined by the conditions of society" [8].

\section{B. Topic Schema}

Topic schema refers to topic-related knowledge. It can be a complex schema of "environment protection" which may consist of a lot more sub-schemata, or a much simpler schema of "Deng Yaping" being a ping-pong player. In interpreting it takes more than merely language schemata to understand the source text and produce the target text. Topic schemata are a must for comprehension and reformulation. In order to understand what is being said during the comprehension phase, the interpreter must have sufficient topic schemata. For example, an interpreter attending a symposium on cardiovascular surgery is supposed to have some topic schemata related to cardiovascular surgery so that he or she can understand the speeches delivered by the medical experts attending the symposium and make himself or herself understood in reformulation. Lack of topic schemata will result in comprehension and reformulation problems and even complete failure in interpretation.

\section{Text Schema}

Text schema refers to the knowledge of text, which is defined by Halliday and Hason as "any passage, spoken or written, of whatever length, that does form a unified whole" [9] The text schema offers answers to how cohesion and coherence are achieved in a text, how information is organized to serve different text purposes, how the language is used stylistically, and so on. It plays an important part in comprehension and memorizing in interpreting in that it offers the interpreter the clue to retrieving and anticipating information as well.

Concerning interpretation, it can be stated beyond doubt that texts with a high proportion of schematically organized text elements may be handled by the interpreter with a minimum of cognitive effort. When texts are dominated by novel features for which no schematic transfer routines are available, both text recognition and text reproduction may exert great mental demand on the interpreter.

\section{Cultural Schema}

Cultural schema refers to culture-specific schemata shared only by people of a certain culture rather than shared universally. Cultural schema may include culture-specific expressions, norms, customs, values, feelings towards certain images and acts, etc. A schema can be neutral in one culture but culture-specific in another. Suppose a Chinese student who has a quite good command of English, reads the famous poem "Ode to West Wind" by Shelley, he can't fail to know the literal meaning of "west wind", but the schema of warm breeze like "east wind" in China will never be activated. Thus here the schema of "west wind" can be seen as a cultural schema specific to the British but only a language schema to Chinese. 


\section{E. Strategy Schema}

It is agreed in the circle of interpreting studies that a competent interpreter is necessarily bilingual but a bilingual is not necessarily a competent interpreter. Why? The strategy schema is the answer. A bilingual may have as good a command of all the four types of schemata discussed above as that of a competent interpreter, but without strategy schemata, which are built up by an interpreter through training and practice, he or she can not hope to bring about successful interpretation.

The strategy schema is a kind of control and coping system organized in the network of schemata. The author divides the strategy schema into the control schema and coping schema. The control schema refers to the mechanism which monitors the voice, speech speed, and interpreting errors of the interpreter, reactions and feedback of the speaker and the listener during the whole interpreting process to make adjustments in interpreting where necessary, make decisions on what coping strategies and interpreting skills to employ. Through monitoring and decision making, the control schema ensures a coordinated utilization of the mental efforts by deploying the other schemata efficiently. As for the coping schema, it refers to coping strategies in interpretation, such as coping strategies to overcome schema gaps, coping tactics like skipping, anticipation, reconstruction, abstraction, and interpreting skills like note-taking, public-speaking, and behaving professionally.

\section{A SCHEMA MODEL FOR INTERPRETING PROCESS}

The author, on the basis of the previous analysis of the five types of schema, puts forward a schema model for interpreting process.

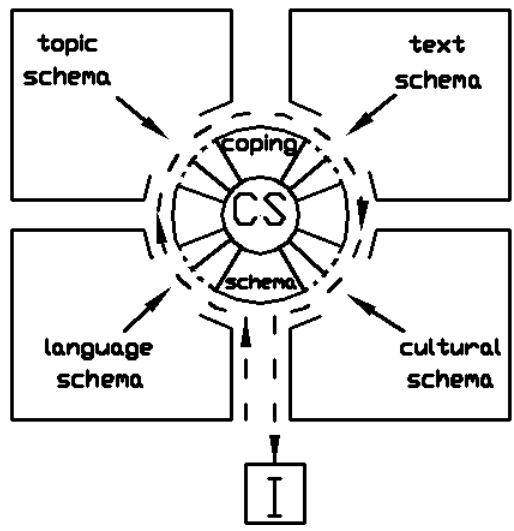

Fig. 1 A Schema Model for Interpreting Process (I refers to interpreting)

The circle in the center refers to strategy schemata with the control schema at the core (represented as CS in the figure). The strategy schema consists of the control schema and coping schema, which refers to various coping strategies and interpreting skills conducive to successful interpreting performance.

The other four types of schemata are shown in the model as being of equal size so as to look good, while in fact these schemata may be of different size from interpreter to interpreter. An interpreter's language schemata may be of bigger size than that of topic schemata. His or her cultural schemata and text schemata, whose importance many interpreters fail to recognize, may even be much smaller.

There is an opening in each schema area. The author calls it "the gate" in a figurative way. The wider "the gate" is, the more efficiently an interpreter is able to employ the correspondent schemata. It goes without saying that every interpreter hopes to have, or rather, is required of, a wide "gate". Then he or she has to build up more coping schemata which, in the author's viewpoint, help to make "the gate" wide. Without them, "the gate" can be narrow. Or in other words, the interpreter needs them to make efficient use of the other four types of schemata.

The five types of schema work together to help bring about successful interpretation. For example, in English-Chinese consecutive interpretation, the sender encodes a message on the basis of the schemata in his long-term memory, and sends the message out. The interpreter, with the phonological schema activated by the sound stimulus, matches the sound with a word, for example, in a speech about IT, matching the sound with the word "memory" (this process is influenced by the interpreter's listening comprehension and the senders' accents), and then seek in the topic schemata for its meaning. If there is the topic schema of "memory" meaning "neicun" available, it will be activated to decode "memory" into "neicun"; otherwise, the interpreter has to make best use of his semantic schemata, while relying on related topic schemata, to generate a temporary translation for this word, such as "jiyiti" . Maybe such a translation is unavailable due to time pressure, the control schema will decide whether just to translate it into "jiyi" or ask for help or even omit it. In this process the phonological, semantic schemata and topic schemata work together on meaning determination of words and also on the decision-making of whether an explanation is needed to offer background information to topic-related expressions that the listener may not be familiar with.

\section{EFFICIENT AND COORDINATED SCHEMATIC DEPLOYMENT IN THE INTERPRETING PROCESS}

From the explanations of the schema model above, it can be seen that in the interpreting process, an efficient and coordinated use of the five types of schema takes place to make interpretation successful. A single type of schema alone, such as the language schema, is often not enough for the interpreter to fulfill an interpreting task.

In the interpreting process the processing capacity of an interpreter is only in limited supply which can be referred to as buffer. The capacity of buffer is limited and differs from interpreter to interpreter, although it can be enlarged through training and practice. When buffer is fully taken up, new information can't be sent to the buffer to be processed, and information will be lost. When buffer is fully taken up, mistakes may occur in comprehension, memory and production. To achieve a coordinated buffer distribution, an efficient and coordinated deployment of the five types of schema is required.

As discussed previously, the strategy schemata is divided into the control schema and coping schema. The control 
schema is responsible for the coordinated distribution of buffer by ensuring an efficient and coordinated deployment of the five types of schema. Such a function is realized through monitoring the whole process and making decisions on what coping schema to employ. For example, in consecutive interpretation, if the interpreter is familiar with the topic and text type used by the speaker, the control schema may decrease buffer in note-taking but distribute more buffer to memorizing the original information on the basis of comprehension; on the other hand, if the interpreter is not familiar with the topic and text type, the control schema will increase the note-taking buffer. It can be seen that such a decision on how much to take down is dependent on the interpreter's topic schemata and text schemata. The control schema just plays the role of deploying the schemata in an efficient and coordinated way. As for coping schemata, they are deployed by the control schema to make best use of the other four schemata. For example, in the case of the illogical ordering of wordy information on the speaker's part, if the interpreter has strong language schemata and text schemata as well, the control schema may deploy the abstraction coping schema to bring about a neat interpretation rather than resort to word-for-word and sentence-for-sentence rendering. In a word, the control schema, with all the other four types of schema and the coping schema at its disposal, makes an efficient and coordinated deployment of the other four types of schema through appropriate employment of coping schemata by monitoring the interpreting process.

\section{CONCLUSION}

Interpreting is a complicated multi-tasking processing activity. According to Gile [1], the mental efforts of listening comprehension, memory and production compete for allocation of limited processing capacity, resulting in information loss, distortion in the case of overload. And hence a well balanced, reasonable distribution of attention is thought to be of vital importance to an interpreter. The schematic approach in this regard offers some insights. The schema model for interpreting process put forward here illustrates how schemata work together to ensure successful interpretation. It is clear that an efficient deployment of schemata helps achieve coordination of the mental efforts with the control schema at the core to monitor the whole interpreting process and make strategic decisions on what coping schemata to activate to cope with difficulties met in the interpreting process and better balance the mental effort load. The analysis shows that schemata play a very important role in comprehension, memory, and production.

It takes the interaction and coordination of the five types of schema to achieve successful interpreting. An interpreter or an interpreter-to-be, if he or she hopes to be a competent interpreter, should strive to improve his or her schematic competence, which refers to the quantity and scale of his or her schemata, and efficient employment of schemata in the interpreting process.

\section{REFERENCES}

[1] Gile, D. Basic Concepts and Models for Interpreter and Translator Training [M]. Amsterdam/Philadelphia: John Benjamins Publishing Company, 1995.

[2] Bartlett, F.C. Remembering [M]. London: Cambridge University Press, 1932.

[3] Minsky, M. A Framework for Representing Knowledge. In The Psychology of Computer Vision [C]. ed. Winston, P.H. New York: Mc Graw-Hill, 1975.

[4] Mckeown, K.R. Text Generation [M]. Combridge: Combridge University Press, 1985.

[5] Carrel, P.L. Three Components of Background Knowledge in Reading Comprehension [J]. Language Learning, 1983, 33 (1): 83-203.

[6] Cook, G. Discourse and Literature [M]. Oxford: Oxford University Press, 1995.

[7] Asher, R. E. et al eds. The Encyclopedia of Language and Linguistics [Z], Vol. 4. Oxford: Pergamon Press, 1994.

[8] Mey, J. Pragmatics: An Introduction [M]. Beijing: Foreign Language Teaching and Research Press, 2001.

[9] Halliday, M. A. K. and Hasan, R. Cohesion in English [M]. Beijing: Foreign Language Teaching and Research Press, 2001. 